\title{
Mangelernährung - auch in der Schweiz ein Problem
}

\author{
Mangelernährung kann bei Personen mit Unter-, Normal- und Übergewicht \\ vorkommen. Die Diagnose wird bei jedem fünften hospitalisierten Patienten in der \\ Schweiz festgestellt. Die neue Broschüre «Mangelernährung trotz Überfluss?!», \\ gemeinsam verfasst von Fachorganisationen und Berufsverbänden, informiert über \\ diese alarmierende Situation.
}

Reinhard Imoberdorfa, Maya Rühlin ${ }^{b}$, Rémy Meierc, Peter E. Ballmer ${ }^{a}$

a Klinik für Innere Medizin, Kantonsspital Winterthur

b Ernährungsberatung, Kantonsspital Winterthur

c Gastroenterologie, Kantonsspital Liestal

Korrespondenz:

Dr. med. Reinhard Imoberdorf Departement Medizin

Klinik für Innere Medizin

Kantonsspital Winterthur

CH-8401 Winterthur

Tel. 0522662121

Fax 0522664706

reinhard.imoberdorf[at]ksw.ch

\section{Mangelernährung? Ja, auch in der Schweiz ein Thema!}

Bei Spitaleintritten werden bei jedem fünften Patienten in der Schweiz Anzeichen von Mangelernährung festgestellt. Dieser Umstand mag erstaunen, bringen doch 37\% der Schweizer Bevölkerung zu viel Gewicht auf die Waage - und Lebensmittel sind in unserem Land in grosser Fülle leicht zugänglich. Die Broschüre «Mangelernährung trotz Überfluss?!» orientiert das breite Publikum über Fakten und räumt Vorurteile aus dem Weg. Sie zeigt auch, dass nicht nur Untergewichtige mangelernährt sind. Der Leitfaden wird unter Regie der Schweizerischen Gesellschaft für Ernährung (SGE), der Gesellschaft für klinische Ernährung in der Schweiz (GESKES) und dem Schweizerischen Verband dipl. Ernährungsberater/innen HF/FH (SVDE) herausgegeben. Realisiert werden konnte die Broschüre dank finanzieller Unterstützung von der Vereinigung Diät -

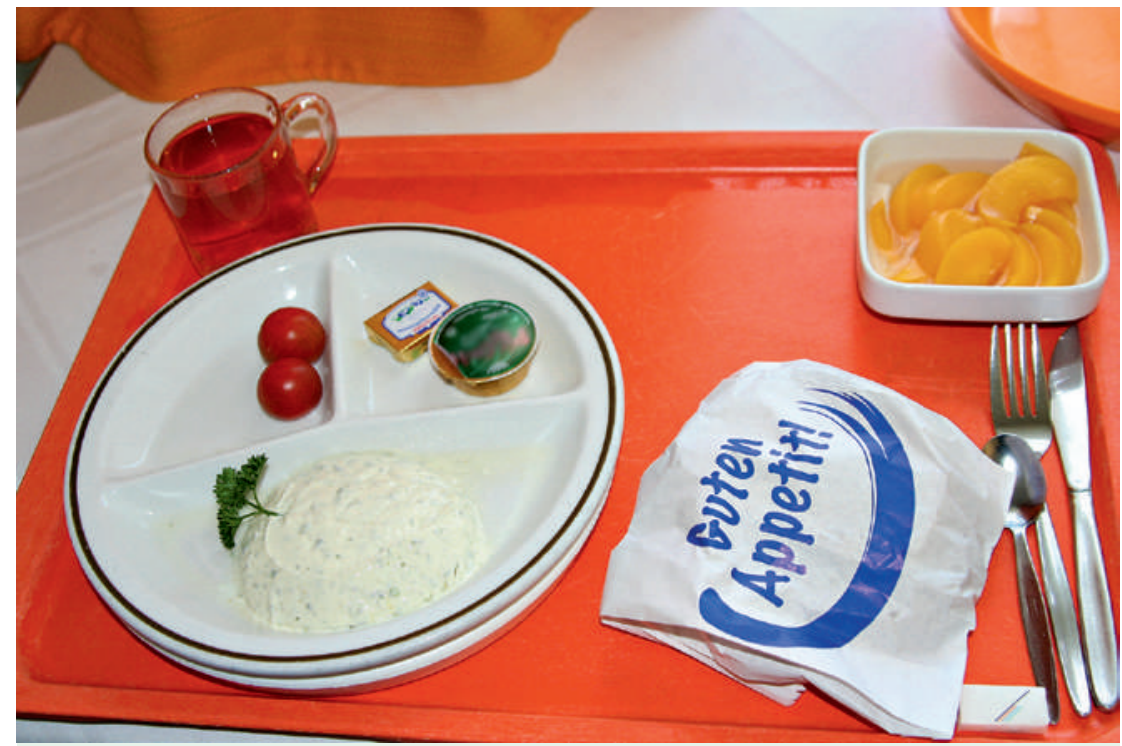

Schon bei Spitaleintritt werden bei jedem fünften Patienten in der Schweiz Anzeichen von Mangelernährung festgestellt.

\section{La dénutrition est aussi un problème en Suisse}

La dénutrition est un problème important et fréquent que l'on rencontre tant au cabinet médical qu'à l'hôpital, en particulier chez les patients âgés. En cas de maladie, elle représente un facteur de risque indépendant qui entraîne une augmentation de la morbidité et de la mortalité, prolonge l'hospitalisation et contribue à l'augmentation des coûts dans le domaine de la santé.

Fachgruppe Medizinische Ernährung und dem Bundesamt für Gesundheit (BAG).

Mangelernährt können Personen mit Unter-, Normal- und Übergewicht sein, wenn eine ausgewogene und bedarfsdeckende Ernährung nicht gewährleistet ist. Zu geringes oder zu einseitiges Essen, krankheitsbedingte Nährstoffverluste oder ein erhöhter Bedarf sind Faktoren, welche die Mangelernährung begünstigen. Die Versorgungsdefizite manifestieren sich schleichend, oft als verborgener Prozess und als Folge von Erkrankung, Schmerzzuständen, Beweglichkeitseinschränkungen, Depression oder Einsamkeit. Aber auch zu hohe Arbeitsbelastung, Stressfaktoren, häufiger Alkoholkonsum, ungesunde Körperideale und Modetrends können Mangelernährung begünstigen. Die Folgen davon sind gestörte Funktionen wichtiger Organe, ein geschwächtes Abwehrsystem und eine verschlechterte Lebensqualität. (Für eine ausführliche Diskussion der medizinischen Bedeutung der Mangelernährung verweisen wir auf den Fortbildungsartikel im Schweizerischen Medizin-Forum [1].)

Die Broschüre verfolgt den Zweck, das Problem der Mangelernährung auf breiter Basis in der Bevölkerung zu thematisieren, mit dem Ziel, Mangeler- 
nährung frühzeitig zu erkennen und entsprechend adäquat zu bekämpfen. Die Broschüre beinhaltet unter anderem einen kurzen Selbsttest und ist für die breite Bevölkerung, die ärztliche Praxis und das Spital als Informationsmaterial geeignet. Über den Bezug der Broschüre finden sich Informationen im Kasten.

\section{Mangelernährung auch im Spital - ein europaweites Problem}

Ein Expertenbericht des Europarates [2] zeigte klar, dass Unterernährung (bzw. Protein-Energie-Mangelernährung) bei hospitalisierten Patienten ein real existierendes Problem in den Krankenhäusern aller europäischer Länder darstellt. Um diese Hürden zu überwinden, appellierte der Europarat an die Verantwortlichkeit der einzelnen Länder.

\section{Der Blick vor die eigene Türe}

Durch den Expertenbericht des Europarates erhielt das Thema Mangelernährung des Spitalpatienten zusätzlich eine politische Dimension, auch für die Schweiz. Wir haben in einer grossen Schweizerischen Untersuchung die Prävalenz der Mangeler-

\section{Nicht nur Untergewichtige sind mangelernährt.}

nährung untersucht [3]. Bei insgesamt 32837 Patienten (16540 Frauen, 16297 Männer) fand man bei 5978 (18,2\%) eine Mangelernährung bereits beim Eintritt ins Spital. Die Prävalenz der Mangeler- nährung war direkt altersabhängig: <45 Jahre: 8\%; 45-64 Jahre:11\%; 65-84 Jahre: 22\%; >85 Jahre: 28\%.

\section{Ökonomische Bedeutung der Mangel- ernährung}

Andreas Frei hat in einer durch das Bundesamt für Gesundheit in Auftrag gegebenen Studie [4] die Kosten für die Mangelernährung in der Schweiz auf durchschnittlich 526 Millionen Schweizer Franken pro Jahr beziffert, und dies bei Gesundheitskosten von über 50 Milliarden im Jahr 2006 für die ganze Schweiz. Gemäss seiner Analyse sind Interventionen zur Erkennung und Behandlung der Mangelernährung verfügbar, wirksam und insgesamt kostensparend. Kosteneinsparungen von 1400 bis 2800 Schweizer Franken bei einem Aufwand von wenigen hundert Franken pro Patient und Spitalaufenthalt erscheinen realistisch.

\section{Literatur}

1 Imoberdorf R, Rühlin M, Beerli A, Ballmer PE. Mangelernährung - Unterernährung. Schweiz Med Forum. 2011;11(44):782-6.

2 Beck AM, Balknäs UN, Fürst P, Hasunen K, Jones L, Keller U, et al. Food and nutritional care in hospitals: how to prevent undernutrition - report and guidelines from the Council of Europe. Clin Nutr. 2001;20(5):455-60.

3 Imoberdorf R, Meier R, Krebs P, Hangartner PJ, Hess B, Stäubli M, et al. Prevalence of undernutrition on admission to Swiss hospitals. Clin Nutr. 2010;29: 38-41.

4 Frei A. Mangelernährung im Spital - medizinische Kosten und Kosteneffektivität bei Verhinderung. Bericht im Auftrag des BAG. (www.bag.admin.ch/ themen/ernaehrung_bewegung/05207/05226/index. html?lang=de).
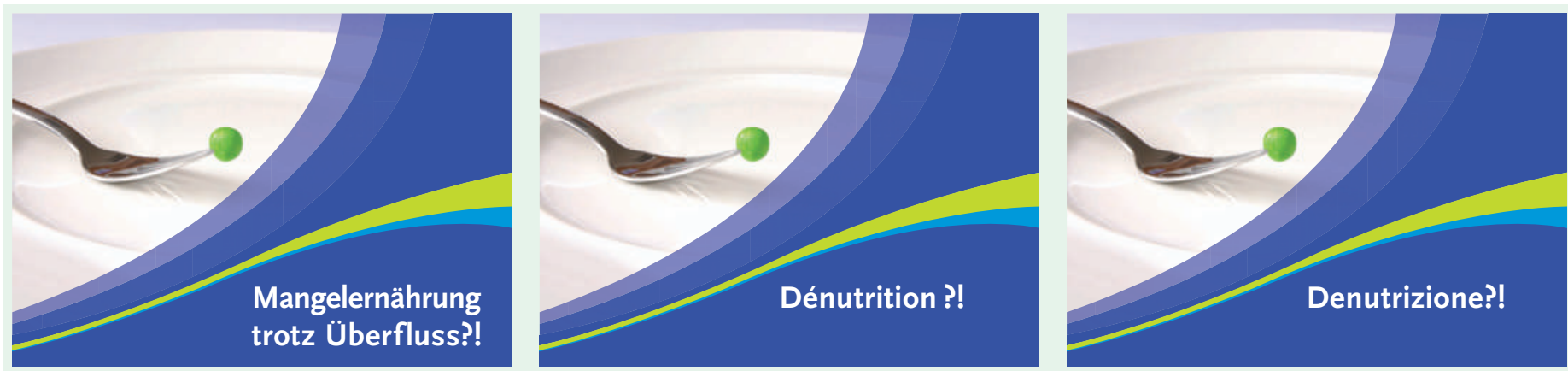

Die Broschüre kann kostenlos bestellt werden bei: Vereinigung Diät, Fachgruppe Medizinische Ernährung, Elfenstr. 19, Postfach 1009, 3000 Bern 6, Fax 0313521185 und E-Mail: silvia.eggimann[at]h-e.ch

Bitte geben Sie die gewünschte Anzahl Broschüren (deutsch, französisch und/oder italienisch) sowie die genaue Zustelladresse an. 